\title{
Axonal loss and neuroinflammation caused by peroxisome-deficient oligodendrocytes
}

\author{
Celia M Kassmann ${ }^{1}$, Corinna Lappe-Siefke ${ }^{1}$, Myriam Baes ${ }^{2}$, Britta Brügger ${ }^{3}$, Alexander Mildner ${ }^{4}$, \\ Hauke B Werner ${ }^{1}$, Oliver Natt ${ }^{5}$, Thomas Michaelis ${ }^{5}$, Marco Prinz ${ }^{4}$, Jens Frahm ${ }^{5}$ \& Klaus-Armin Nave ${ }^{1}$
}

Oligodendrocytes myelinate axons for rapid impulse conduction and contribute to normal axonal functions in the central nervous system. In multiple sclerosis, demyelination is caused by autoimmune attacks, but the role of oligodendroglial cells in disease progression and axon degeneration is unclear. Here we show that oligodendrocytes harbor peroxisomes whose function is essential for maintaining white matter tracts throughout adult life. By selectively inactivating the import factor PEX5 in myelinating glia, we generated mutant mice that developed normally, but within several months showed ataxia, tremor and premature death. Absence of functional peroxisomes from oligodendrocytes caused widespread axonal degeneration and progressive subcortical demyelination, but did not interfere with glial survival. Moreover, it caused a strong proinflammatory milieu and, unexpectedly, the infiltration of $B$ and activated $\mathrm{CDB}^{+} \mathrm{T}$ cells into brain lesions. We conclude that peroxisomes provide oligodendrocytes with an essential neuroprotective function against axon degeneration and neuroinflammation, which is relevant for human demyelinating diseases.

The best known function of oligodendrocytes in the central nervous system (CNS) is the formation of myelin for axonal insulation and rapid impulse propagation ${ }^{1}$. However, there is growing evidence that intact myelination is also essential for the integrity of axons in white matter tracts. Indeed, progressive axonal loss has been recognized as the major cause of clinical impairment in the late stages of human multiple sclerosis ${ }^{2,3}$.

We have previously shown, using gene targeting in mice, that oligodendrocytes maintain the axonal integrity independent of myelination, as the two functions can be genetically uncoupled ${ }^{4,5}$. Also, in the peripheral nervous system, Schwann cell support is required to prevent axonal loss in demyelinating neuropathies ${ }^{6}$. The molecular mechanisms of axonal preservation by glial cells are completely unknown, however, and are likely to include a number of essential axon-glia interactions.

In the CNS, premature axon loss is preceded by abnormal swellings ${ }^{4}$ and is associated with reduced rates of retrograde axonal transport ${ }^{7}$. These pathological features resemble those of mice with mitochondrial impairments ${ }^{8}$, suggesting that an underlying energy failure in axons is caused, at least to some extent, by impaired glial support. We have hypothesized that one physiological function of myelinating oligodendrocytes, specifically in regions of high-level electrical activity and energy consumption, is the metabolic support of axons and the detoxification of reactive oxygen species (ROS) of white matter in order to preserve mitochondrial energy generation.
An important organelle for both detoxification of ROS and fatty acid metabolism is the peroxisome $e^{9,10}$. Peroxisomes are present in virtually all cell types, where they detoxify reactive oxygen species, synthesize plasmalogens and carry out $\beta$-oxidation of fatty acids, specifically of very long chain fatty acids (VLCFA). Whether peroxisomes are essential for myelination is unknown. Human patients with a peroxisome biogenesis defect may survive to show leukoencephalopathy, but dysmyelination is difficult to distinguish by magnetic resonance imaging (MRI) from early demyelination. The corresponding mouse models of Zellweger syndrome die perinatally ${ }^{11}$.

Among the different cell types of the brain, oligodendrocytes have the highest capacity for ROS detoxification ${ }^{12}$. By immunostaining and confocal analysis of adult mouse brains, we noticed that the majority of peroxisomes in white matter tracts are associated with oligodendrocytes rather than with axons (see below). To study the function of these peroxisomes in myelinogenesis and axonal preservation, we inactivated the mouse peroxin-5 (Pex5) gene in oligodendrocytes. PEX5, the peroxisomal targeting signal type-1 receptor, is a cytosolic cycling adaptor essential for importing the majority of proteins into the matrix ${ }^{13}$. Null mutations of this gene are lethal in patients with Zellweger syndrome as well as in mice ${ }^{11,14}$. Here we show that peroxisomes serve essential functions in oligodendrocytes for axonal neuroprotection and maintenance of a noninflammatory environment. We also suggest a model that links this observation to the unknown etiology of X-linked adrenoleukodystrophy.

\footnotetext{
${ }^{1}$ Department of Neurogenetics, Max Planck Institute of Experimental Medicine, Hermann-Rein-Strasse 3, D-37075 Göttingen, Germany. ${ }^{2}$ Laboratory for Cell Metabolism, Faculty of Pharmacy, Katholieke Universiteit Leuven, 3000 Leuven, Belgium. ${ }^{3}$ Biochemie-Zentrum, Universität Heidelberg, D-69120 Heidelberg, Germany. ${ }^{4}$ Department of Neuropathology, University of Göttingen, D-37075 Göttingen, Germany. ${ }^{5}$ Biomedizinische NMR Forschungs GmbH am Max-Planck-Institut
} für biophysikalische Chemie, 37077 Göttingen, Germany. Correspondence should be addressed to K.A.N. (nave@em.mpg.de). 
a
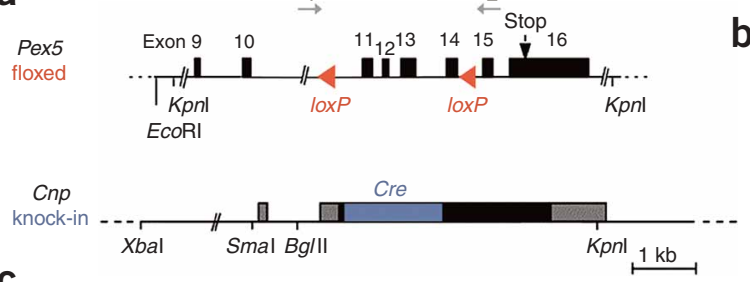

C Control

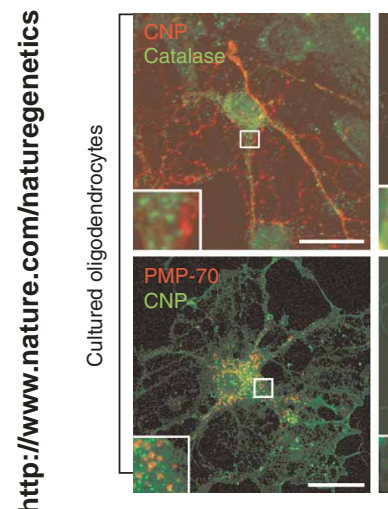
Mutant
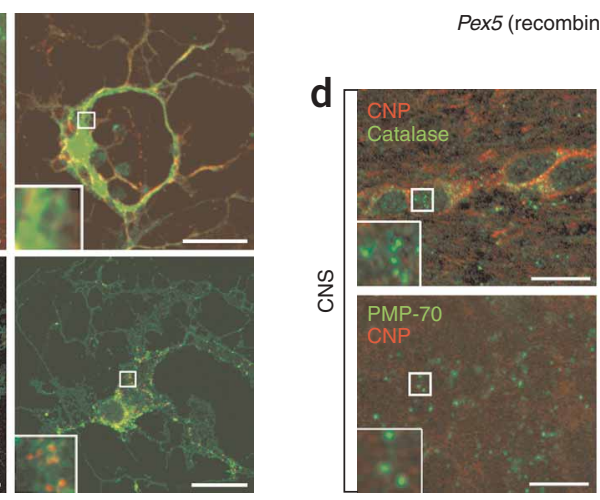

을 Figure 1 Inactivation of peroxisome function in oligodendrocytes. (a) Structure of the floxed (red) Pex5 gene (top) and the Cnp (Cre knock-in, blue) locus (bottom). Primers 1 and 2 (gray arrows) were used to demonstrate genomic recombination. (b) Genotyping PCR using brain-derived DNA (top, middle) and PCR proving genomic recombination (bottom), using genomic DNA obtained from a homozygous Pex5 floxfflox control mouse ('flox control'), a homozygous Cnp-Cre/Cre mouse ('Cre control') and a Pex $5^{f l o x} / f l o x * C n p-C r e /+$ mutant mouse ('mutant'). WT, wild type. Primers 1 and 2 amplify a 600-bp fragment specific for the recombined Pex5 allele (right lane). For genotyping primers see refs. 5,15. (c,d) Confocal images of CNP-stained oligodendrocytes in primary culture (c) and in histological sections at the age of 2 months (d), obtained from control mice (left) and Pex5 mutants (right). Punctate distribution of catalase (in green, magnified in inset) demonstrates intact peroxisomes (top left). Mutant oligodendrocytes are diffusely stained (top right). In contrast, PMP-70 (magnified in inset) labels peroxisomal 'ghosts' in mutant oligodendrocytes. Most catalase ${ }^{+}$puncta (green) within the white matter are associated with CNP-stained oligodendrocytes (d, top left). Note that the overall expression level of catalase is increased in mutant oligodendrocytes. Scale bars, $20 \mu \mathrm{m}$ in c; $10 \mu \mathrm{m}$ in d.

\section{RESULTS}

\section{Targeting peroxisome functions in oligodendrocytes}

By cross-breeding a mouse line in which Pex5 exons 11 to 14 are flanked by loxP sequences ${ }^{15}$ ('floxed') with mice expressing Cre recombinase under control of the $2^{\prime}, 3^{\prime}$-cyclic nucleotide phosphodiesterase gene $(C n p)$ promoter ${ }^{5}$, we obtained mutant mice with Pex5 inactivation restricted to myelin-forming glia (Fig. 1a). These conditional mutants were born at the expected mendelian frequency. In the following, we refer to Pex $5^{\text {flox/flox*}} \mathrm{Cnp}$-Cre/+ mice as 'mutants', and to $P e x 5^{f l o x /+}$ mice (with and without a Cnp-Cre allele) and Pex $5^{\text {flox/flox }}$ mice as 'controls' phenotypically indistinguishable from one another. We confirmed Cre-mediated recombination PCR analysis of brain genomic DNA, using primers $300 \mathrm{bp}$ upstream (sense) and $300 \mathrm{bp}$ downstream (antisense) of the 2-kb floxed region, yielding the expected 600-bp fragment (Fig. 1b, Supplementary Fig. 1a online). Cell-specific recombination and loss of PEX5 function was studied in dissociated cells derived from mutant brains and in brain sections. In mutant oligodendrocytes, identified by staining for CNP, catalase was localized in the cytosol, in contrast to control oligodendrocytes that showed catalase associated with (peroxisomal) puncta (Fig. 1c,d; upper panels). The peroxisomal membrane protein PMP-70 had a punctate distribution in both control and mutant oligodendrocytes (Fig. 1c,d; lower panels). Thus, import of peroxisomal matrix proteins was functionally disrupted in mutant oligodendrocytes, leaving

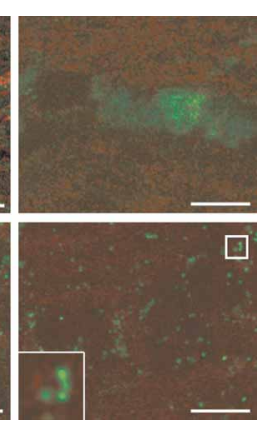

$\mathrm{PMP}^{+}$peroxisomal 'ghosts', and these were less abundant than normal peroxisomes. Peroxisomal ghosts are similarly decreased in abundance in fibroblasts from PEX5-deficient humans with Zellweger syndrome ${ }^{16}$. We also confirmed oligodendrocyte-specific Cre expression in vivo by immunostaining of 7-d-old and adult mice. In subcortical white matter, almost all oligodendrocytes expressed the transgene (Supplementary Fig. 1b and data not shown), in agreement with earlier studies ${ }^{17}$. Also histologically, we found the peroxisomal import defect of catalase in mutant oligodendrocytes (Fig. 1d). In contrast, the somas of all other cells in the CNS showed a punctate (peroxisomal) staining. In costainings with catalase and CNP, most identifiable peroxisomes within white matter tracts were associated with oligodendrocytes and lost in Pex5 mutant mice (data not shown).

\section{Peroxisomal lipid metabolism}

Purified myelin from mutant mice showed specific biochemical abnormalities. Whereas the stoichiometry of major myelin lipids was preserved (data not shown), plasmalogens were reduced by $80 \%$ (and phosphatidylethanolamines correspondingly increased) (Fig. 2a,b $)^{18}$. As plasmalogen biosynthesis relies on dihydroxyacetone-phosphate acyltransferase, a peroxisomal enzyme ${ }^{19}$, this demonstrates that targeting the Pex5 gene impaired the peroxisomal functions in oligodendrocytes in vivo also.

The accumulation of VLCFA is a biochemical hallmark of peroxisomal disorders in humans ${ }^{20,21}$. By gas chromatography and mass spectrometry, we determined the C26:0 to C22:0 ratio of fatty acids in mutant mice (Fig. 2c). As expected, there was a significant increase of VLCFA in purified myelin at age 2 months (threefold elevation) and age 11 months (eightfold). Unexpectedly, however, we found a similar increase of VLCFA in whole brain lysates (threefold and sixfold, respectively). Indeed, the accumulation of VLCFA caused by the loss of peroxisomes in oligodendrocytes is comparable to that found earlier $^{22-24}$ (fourfold elevation) in brain lysates from $A b c d 1$ null mice. Taken together, these findings imply that oligodendroglial peroxisomes are largely responsible for the degradation of VLCFA in the adult brain.

\section{Progressive axonal loss and demyelination}

Notably, conditional Pex5 mutants showed no obvious developmental delay or a neurological defect indicative of CNS dysmyelination (we cannot formally exclude the possibility that 'normal' development reflects a temporal delay between Cre recombination and peroxisome dysfunction in vivo). Up to the age of 2 months, myelin sheaths were morphologically normal, as assessed by light microscopic and electron microscopic criteria (Supplementary Fig. 2 online). At age 3 months, $83.5 \%$ of a cohort of mice was indistinguishable from controls (defined as 'clinical stage I'), whereas $14 \%$ showed hind limb ataxia (stage II) when performing a grid test (Fig. 3a, bottom; 


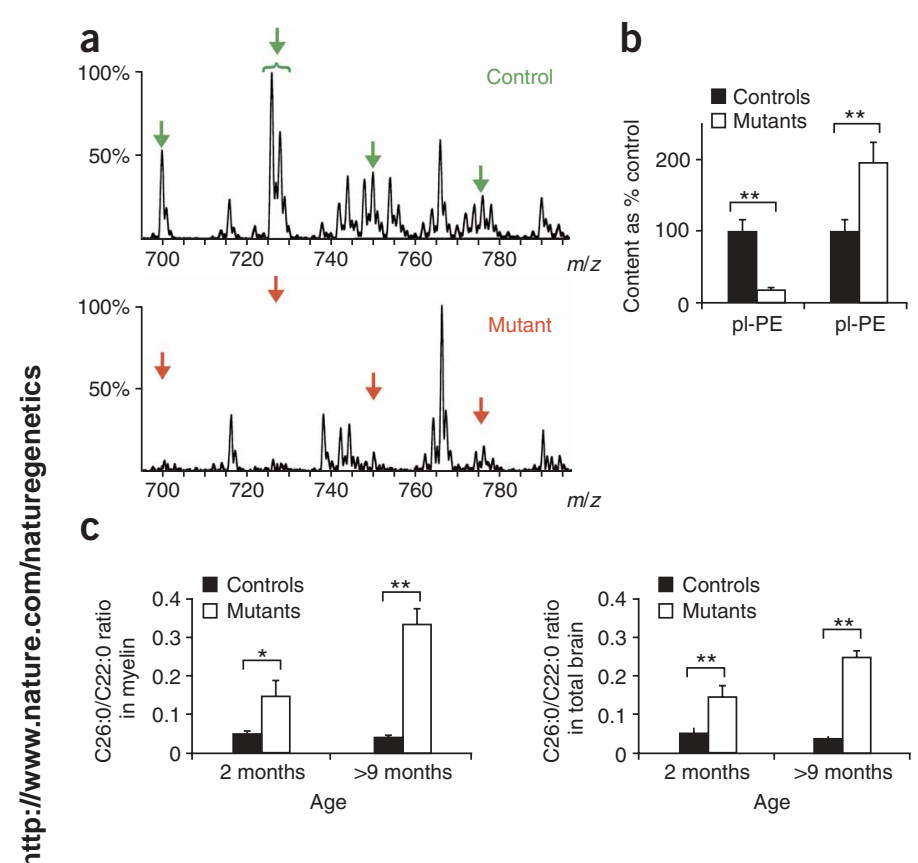

윽 Supplementary Video 1 online). When disease progression was followed over the next 12 months (Fig. 3b-d), we found an increasing percentage of mice showing hunchback (stage III), hindlimb paresis and tremor (stage IV), and finally a paralysis with breathing difficulties (stage V). Interindividual differences were most pronounced at age 6-7 months, when mutants were found at all clinical stages (Fig. 3c). Disease progression was most obvious at age 9 months. At 12 months, $>91 \%$ of all mutants had died (or had to be put to death), and no mutant lived past 13 months (Fig. 3d).

We suspected that mutant mice were developing a late-onset neurodegenerative disease and also followed individual mice by T1- and T2-weighted MRI in vivo. At age 2 months, conditional (2) mutants were indistinguishable from control mice, with a normal white matter (Fig. 4 and Supplementary Fig. 3 online). However, the same mouse at age 4 months (and clinical stage II) showed hyperintense signals in T2-weighted MRI of the rostral subcortical white
Figure 2 Abnormal lipid synthesis and metabolism. (a) Lack of plasmalogens as detected by quantitative mass spectrometry of purified brain myelin lipids from control and Pex5 mutant mice (age 11 months). Arrows indicate the positions of plasmalogens in the spectrograph of a control mouse (top). The same plasmalogens (arrows) are strongly reduced in the Pex5 mutant brains (bottom). (b) Quantification of phosphatidylethanolamine plasmalogens ( $\mathrm{pl}-\mathrm{PE}$ ) and phosphatidylethanolamine (PE), both normalized to protein content, using purified brain myelin from controls and mutants at age 11 months. Values are expressed as a percentage of the controls' mean ( \pm s.e.m.; ${ }^{* *} P<$ $0.005 ; n=4$ or 5 ). (c) Analysis of VLCFA by gas chromatography-mass spectrometry in control mice and Pex5 mutants at ages 2 and 11 months. Values are expressed as mean \pm s.e.m. $\left({ }^{*} P<0.05 ;{ }^{*} P<0.005 ; n=3\right.$ ).

matter, the genu of the corpus callosum (Fig. 4). At that same time, other white matter tracts appeared still intact. In the following months, MRI revealed an extension of white matter abnormalities that reached the hippocampal fimbria (caudally), the anterior commissure (ventrally) and the dorsal corpus callosum. These changes correlated well with clinical deterioration. Notably, even in more severely affected mice (stage IV) the internal capsule, the hippocampal commissure and cerebellar white matter appeared to be spared. T1-weighted MRI yielded corresponding abnormalities (hypointense signal alterations) but was less sensitive (Supplementary Fig. 3b,c).

To determine the underlying pathology, we analyzed horizontal brain sections at different clinical stages. When myelin was visualized by Gallyas silver impregnation, subcortical white matter was normal in 2-month-old mice (stage I) but showed extensive neurodegeneration in older mutants (stage III), with the nearly complete loss of myelinated axons at the final stage (Fig. 5a, Supplementary Fig. 2a). Beginning with stage II, a gradual loss of myelinated fibers was detectable microscopically in the genu of corpus callosum, whereas other cerebral white matter structures were normal (data not shown), in agreement with the MRI data and immunohistochemical staining for proteolipid protein (PLP) (Fig. 5b). To determine whether demyelination was simply the result of oligodendrocyte death, we stained sections with antibodies against Cre (used here as a nuclear marker of live oligodendrocytes) and the oligodendrocyte-specific CNP. This showed normal numbers of oligodendrocytes in newborn and 2-month-old mutants with a characteristic arrangement in rows

Figure 3 Neurological impairments and premature death. (a) The 'bar test' (top), showing hindlimb weakness of mutant mice. Mice developed a kyphosis (red arrowhead). The 'grid test' (bottom) was used to detect subtle motor coordination defects, such as slipping (red arrow). (b) Clinical staging of mutant mice. (c) Assessment of the distribution of clinical stages for different age groups of mutant mice ( $n=79$ ). At age 3 months, $84 \%$ were healthy (stage I, white bars) and $14 \%$ showed motor coordination defects of the hindlimbs (stage II, yellow bars). At 6 months, mutants were found also at stages III (orange bars), IV (bright red bars) and $\mathrm{V}$ (dark red bars). At 12 months, more than $90 \%$ of mutant mice were endstage or had died. (d) Kaplan-Meier survival curve of control mice $(n=173)$ and Pex5 mutants $(n=149)$, showing a $50 \%$ probability of mutant mice reaching the age of 11 months. Note the heterogeneity of the disease course and the maximal life span of $<14$ months on the current non-inbred background.
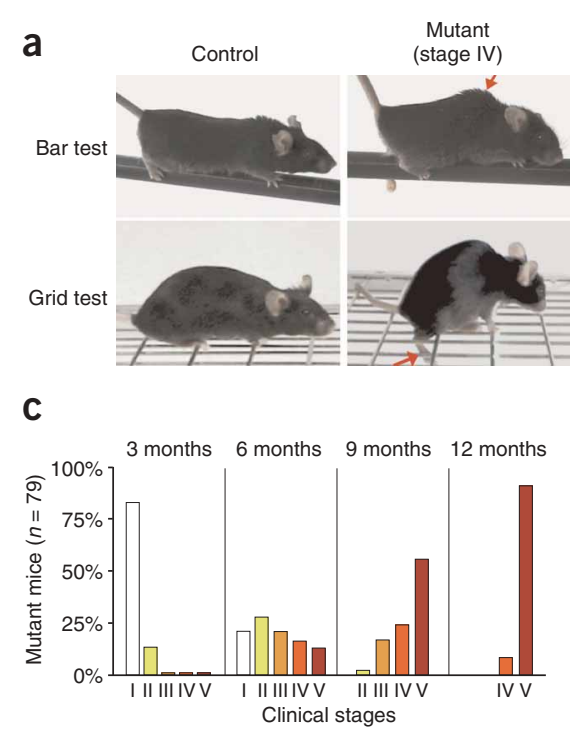

b

\begin{tabular}{ccccccc}
$\begin{array}{c}\text { Behavioral } \\
\text { abnormalities }\end{array}$ & I & II & III & IV & V \\
\hline Hindlimb ataxia & - & + & + & + & + \\
Kyphosis & - & - & + & + & + \\
Hindlimb paresis & - & - & - & + & + \\
Forelimb ataxia & - & - & - & + & + \\
Tremor & - & - & - & + & + \\
Hindlimb paralysis & - & - & - & - & + \\
Passiveness & - & - & - & - & + \\
Breathing difficulty & - & - & - & - & +
\end{tabular}

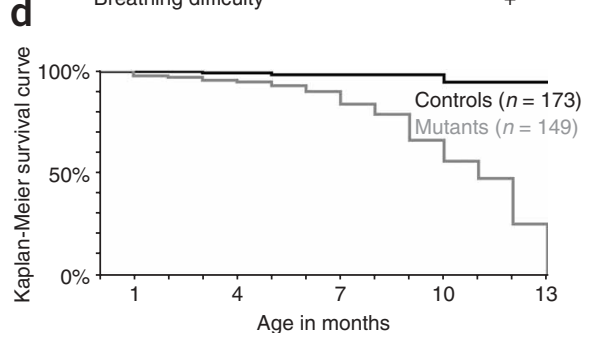



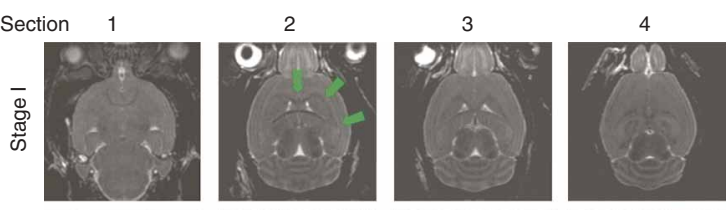

Age
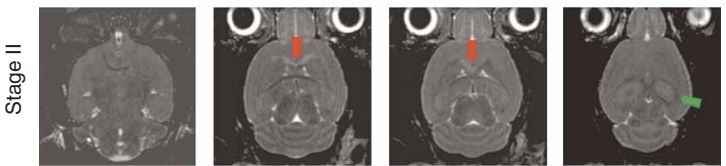

4 months
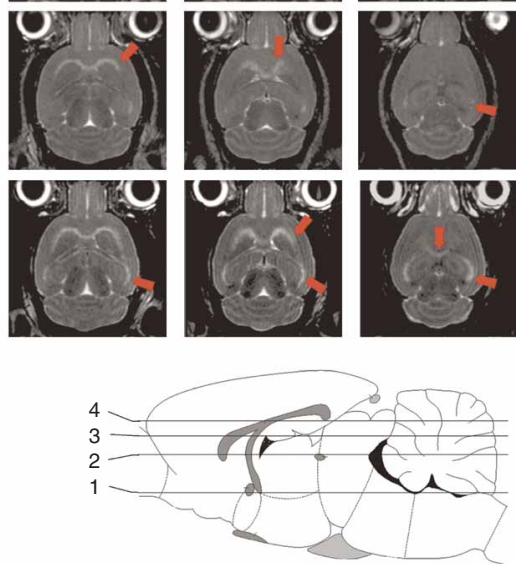

(middle panel in Supplementary Fig. 1b). Oligodendrocytes in older mice were viable, even in severely affected areas (right panel in Supplementary Fig. 1b). TUNEL staining did not show widespread apoptosis, although apoptotic nuclei were occasionally found in affected white matter (Supplementary Fig. 4 online). The identity of these cells was not determined, but cell death did not obviously
Figure $4 \mathrm{MRI}$. T2-weighted MRI of a mouse mutant analyzed at subsequent clinical stages, covering the period from 2 to 8 months of age (stages I-IV; top to bottom). Each row shows four horizontal brain sections from one three-dimensional MRI dataset (1-4, as indicated in schematic brain drawing, bottom). Images from the still-healthy mouse (stage I) showed normal myelin (top, green arrows). Hyperintense signals appeared in the frontal part of the corpus callosum at clinical stage II (second row, red arrows). At clinical stage III, dorsal subcortical white matter also showed hyperintense signals (third row, section 4, red arrow), whereas the anterior commissure remained normal (third row, section 1, green arrow). Signal intensity in white matter structures was increased at stage IV. Additionally, the anterior commissure was abnormal (bottom row, section 1).

precede axonal loss or demyelination. We also noted that the ultrastructure of mitochondria in Pex5 mutant oligodendrocytes was not obviously abnormal (data not shown).

By Bielschowsky silver impregnation or immunostaining of neurofilaments (SMI-31, FNP-7), subcortical axons were intact in young mice (stage I), whereas older mutants (stage III) showed significant axonal loss first observed in the genu of corpus callosum (Fig. 5c and data not shown). In cerebellar white matter (stage $\mathrm{V}$ ), axonal staining was also significantly reduced but demyelination was less pronounced than in the subcortical white matter. Even in younger mutants that showed no obvious demyelination and were clinically stage I, immunolabeling of amyloid precursor protein (APP) already showed axonal swellings in the corpus callosum and in the spinal cord, a sign of disturbances of axonal transport (Fig. 5d). The fact that PEX5deficient oligodendrocytes failed to maintain axonal integrity, even in the absence of visible demyelination, implies a critical function of oligodendroglial peroxisomes for the support of axonal integrity. In corpus callosum, the density of $\mathrm{APP}^{+}$deposits increased over time, but finally decreased in moribund mice, presumably because of complete axonal degeneration (Fig. 5d and data not shown). Even in clinically
Figure 5 Progressive demyelination and axonal

loss in the subcortical white matter. (a) Gallyas silver impregnation of control (left) and mutant brains (stage III, middle; stage $\mathrm{V}$, right) showing progressive decrease of subcortical myelin in adult mice. Boxed area is magnified below. Note that demyelination is less pronounced in the cerebellar white matter than in the corpus callosum. Scale bar, $30 \mu \mathrm{m}$. (b) At higher magnification, progressive loss of myelin is confirmed by immunohistochemical staining for PLP in the corpus callosum of control (left) and mutant brains (middle; stage III; right; stage V). Scale bar, $30 \mu \mathrm{m}$. (c) Axonal loss in the subcortical white matter, revealed by Bielschowsky silver impregnation, beginning in the genu of corpus callosum (middle) and progressing laterally (stage III, middle; stage V, right). Boxed area is magnified below. Note severe axonal loss in cerebellar white matter as well. Scale bar, $30 \mu \mathrm{m}$. (d) Immunohistochemical detection of APP, indicating axonal swellings (arrowhead) that initially appear in the corpus callosum at clinical stage I (middle). At clinical stage III, axonal swellings are more prominent (right; magnified in inset). Scale bar, $30 \mu \mathrm{m}$. (e) Electron micrographs of the CNS white matter showing axonal swellings in mutants at stage $\mathrm{V}$.

Demyelinated axons are frequent (red asterisks). Thin myelin sheaths suggest remyelination (red arrows). Axonal swellings (Sw) were found in demyelinated areas (left) but were more frequent in regions in which myelin is preserved (right), suggesting that axonal swellings precede axon loss and demyelination. Scale bars, $1.1 \mu \mathrm{m}$.

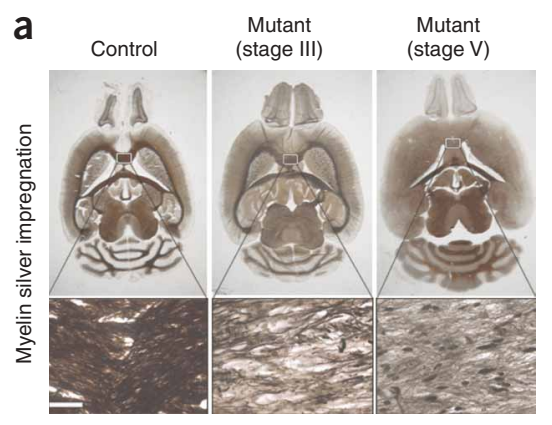

b
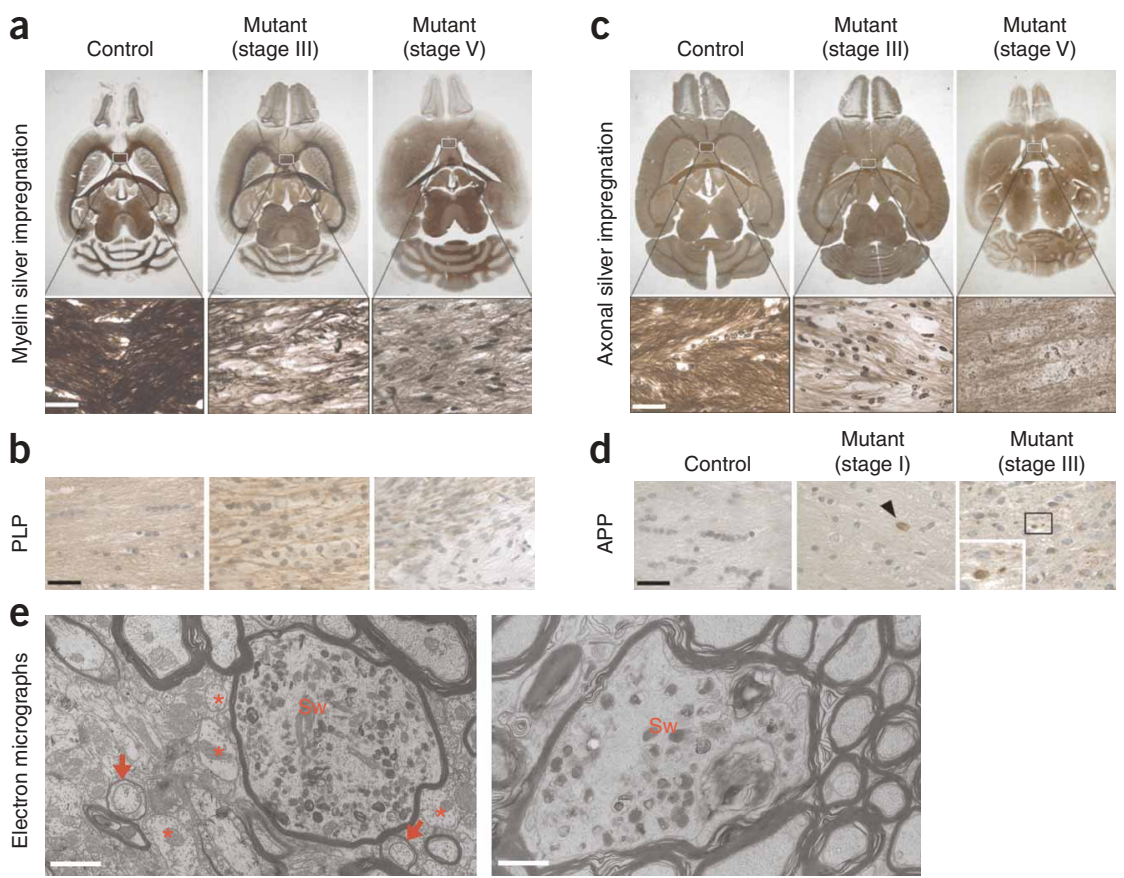

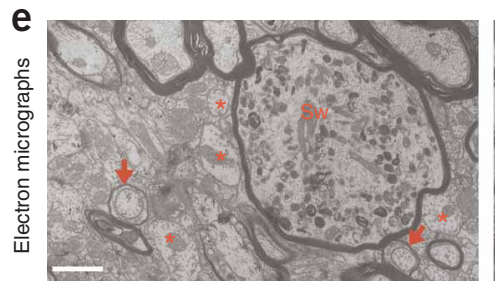




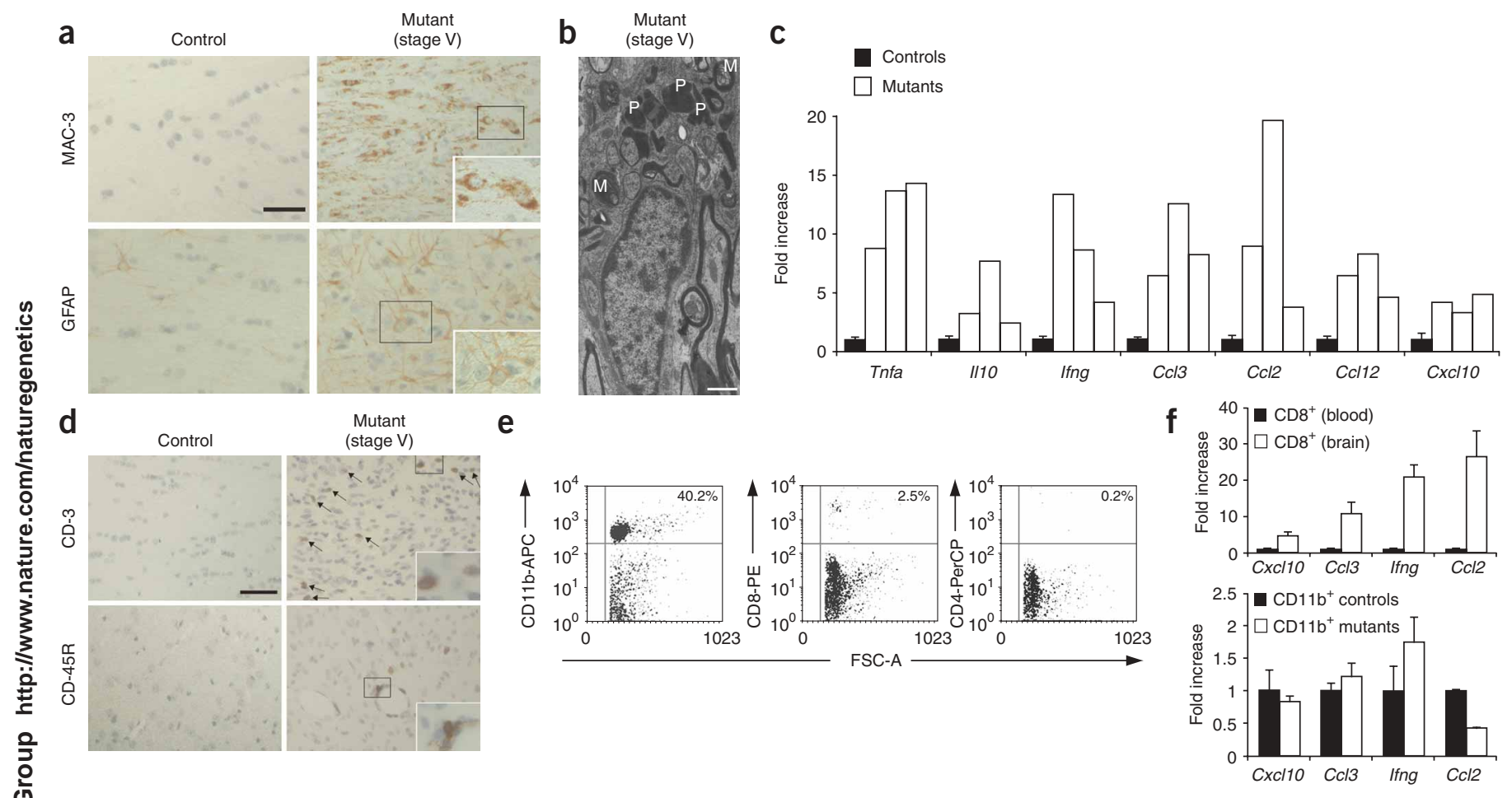

Figure 6 Reactive gliosis and neuroinflammation. (a) Reactive glial cells in the corpus callosum, magnified in insets. Activated microglia or macrophages of mutant mice (right; stage $\mathrm{V}$ ) are immunolabeled by macrophage surface protein MAC-3 (top). Reactive astrocytes are identified by glial fibrillary acidic protein (GFAP, bottom). Scale bar, $30 \mu \mathrm{m}$. (b) Electron micrograph of a macrophage within a lesion, containing several dense-core organelles, presumably peroxisomes (P), and myelin debris (M). Scale bar, $1.1 \mu \mathrm{m}$. (c) By quantitative RT-PCR, proinflammatory cytokines and chemokines are increased in brain cDNA from mutant mice (stage V), when normalized to controls $(n=3$; values \pm s.e.m.). (d) Infiltration of lymphocytes into mutant brains. Detection of (․ T cell receptor protein CD3 reveals T lymphocytes in lesions (arrows in upper right; magnified in inset). B cell-specific phosphatase CD45R shows perivascular B cells (lower right; magnified in inset). Scale bar, $50 \mu \mathrm{m}$. (e) FACS analysis of pooled brain cell suspensions ( $n=6$, age 11 months), prepurified by a Percoll gradient. Left: CD11 b+ monocytes (40.2\%). Middle: CD8 ${ }^{+}$T cells (2.5\%). Right: CD4 ${ }^{+}$T cells $(0.2 \%)$. APC, allophycocyanin; PE, phycoerythrin; peridinin chlorophyll protein; FSC-A, forward scatter area. (f) Left: RT-PCR showing elevated cytokine expression in sorted CD8 ${ }^{+}$T cells from brain relative to $\mathrm{CD} 8^{+} \mathrm{T}$ cells from peripheral blood of the same mutants. Right: cytokine expression in FACS-sorted $\mathrm{CD} 11 \mathrm{~b}^{+}$macrophages or microglia from control and mutant brains.

stage $\mathrm{V}$ mutants, the peripheral nervous system appeared normal at the light-microscopic scale (data not shown). Electron microscopy of subcortical white matter in severely affected mice (stage V) showed myelin sheaths that were stripped off their axons (Fig. 5e), presumably by macrophage activity (see below). When present, myelin sheaths were normal with respect to ultrastructure and periodicity (not shown). Some axons with thin myelin sheaths were indicative of remyelination, and axonal swellings were a frequent ultrastructural finding (Fig. 5e).

\section{Oligodendrocyte dysfunction associated with neuroinflammation} As expected, neurodegeneration was accompanied by gliosis. Using antibodies to MAC3 as a marker of activated microglia and macrophages and to glial fibrillary acidic protein (GFAP) to label astrocytes, we found a massive gliosis in demyelinated areas (Fig. 6a). In mutant mice at stage I, activated microglia or macrophages were the earliest histopathological finding: that is, they were seen before demyelination and axon loss. Astrogliosis could be demonstrated beginning with stage II. Up to stage III, reactive astrocytes and microglia/macrophages were restricted to white matter. Later, at stages IV and V, gliosis had spread to all brain areas. In general, the emergence of activated microglia and astrogliosis preceded demyelination and neurodegeneration in the white matter (as visualized by MRI and histopathology).
Phagocytic activity of microglia/macrophages was indicated by electron microscopy (Fig. 6b) and engulfed CNP-immunoreactive material (data not shown).

In order to determine whether gliosis was accompanied by a change of the CNS homeostasis, we measured the expression of genes encoding proinflammatory cytokines (tumor necrosis factor- $\alpha$ (encoded by Tnfa), interleukin-10 (Il10) and interferon (IFN)- $\gamma$ (Ifng)) as well as chemoattractants for lymphomononuclear cells (MIP-1 $\alpha(C c l 3)$, MCP-1 (Ccl2), MCP-5 (Ccl12) and IP-10 (Cxcl10)). By quantitative RT-PCR at 2, 4, 6 and 10 months of age, we found a marked increase of all proinflammatory factors analyzed, beginning at 4 months (stages II-III); however, there were marked interindividual differences (Fig. $\mathbf{6 c}$ and data not shown).

MCP- 1 and MIP- $1 \alpha$ are mostly produced by astrocytes and macrophages, and have been associated with lymphocyte infiltration in multiple sclerosis ${ }^{25,26}$. This raised the question of whether in Pex5 mutant mice lymphocytes are recruited into demyelinated brain areas. We tested for infiltrating $\mathrm{T}$ cells with antibodies to $\mathrm{CD}-3$ ( $\mathrm{T}$ cell receptor) and for infiltrating $\mathrm{B}$ cells with antibodies to the tyrosine phosphatase CD45R. Notably, we found T cells as early as 4 months of age (stage II), and their numbers increased as disease progressed. T cells were restricted to the lesioned brain areas and not found in normalappearing white matter, indicating that they may follow rather than 
cause demyelination. Their presence was not always accompanied by B cell infiltration; however, we found numerous perivascular B cells (Fig. 6d). About three-quarters of the adult and all of the older mutants showed lymphocyte infiltration, whereas in age-matched controls neither T nor B cells were detected in brain parenchyma or perivascular spaces. Infiltration was not due to unspecific opening of the bloodbrain barrier, as even severely affected mice never showed passage of Evans blue from the circulation into the brain (data not shown).

Next, we used Percoll gradients followed by FACS (or magnetically activated cell sorting from blood) to isolate brain lymphocytes and monocytes from 9-month-old mutants, close to endstage disease, and from controls. Unexpectedly, we found mainly $\mathrm{CD} 8^{+} \mathrm{T}$ cells in the mutant CNS (Fig. 6e, middle) and only a minor fraction of $\mathrm{CD}^{+}$ (including regulatory) $\mathrm{T}$ cells (Fig. 6e, right). $\mathrm{Cd}_{1} 1 \mathrm{~b}^{+}$monocytes (macrophages/microglia) were also abundant (Fig. 6e, left); however, there were too few B cells for further analysis (data not shown). By RTPCR, we found cytokine expression in both monocytes and T cells. Notably, we found elevated levels of mRNAs encoding IP-10, MIP- $1 \alpha$, IFN- $\gamma$ and MCP- 1 in the $\mathrm{CD}^{+} \mathrm{T}$ cells derived from brain, when compared with levels in peripheral-blood $\mathrm{CD}^{+} \mathrm{T}$ cells obtained from the same mice (Fig. 6f, left). Monocytes that were highly abundant in mutant brains showed higher levels of transcripts encoding IFN $-\gamma$ than monocytes from control brains (Fig. 6f, right), but most 윽 cytokine transcripts were not obviously overexpressed at the single-cell level. A more detailed analysis of the invading immune cells will be reported elsewhere.

\section{DISCUSSION}

Genetic inactivation of peroxisomes in oligodendrocytes has provided unexpected insight into the role of a cellular organelle in maintaining the structural and functional integrity of myelinated fibers. By gross histological inspection, axonal degeneration seems to precede demyelination, but the method for detecting loss of axonal integrity (APP staining) is more sensitive than is the method for detecting demyelination of the same axon (myelin staining). We note that no other mutation affecting primarily an oligodendrocyte function, including the absence of the principal myelin structural proteins MBP ('shiverer'), PLP or CNP in mice, has been reported with a similar phenotype with respect to age of onset, the preferential subcortical localization or the extent of axon loss. The degree of inflammation also is unique when compared with that in other mouse mutants ${ }^{27}$, although we find fewer $\mathrm{T}$ cells than in experimental autoimmune encephalomyelitis mice. More importantly, there is no B or $\mathrm{T}$ cell infiltration in PLP-deficient and CNP-deficient mice ${ }^{4,5}$. This demonstrates that inflammation is not unspecific to axonal degeneration or defects of myelin assembly, but is caused by peroxisomal dysfunction in oligodendrocytes and subsequent demyelination. Direct triggers of the inflammation may be lipid breakdown products.

Peroxisomes are ubiquitous organelles, and their predominant association with oligodendrocytes in the white matter of adult mice was unexpected. Based on RT-PCR at postnatal day 7 and CnpCre expression data (this paper and refs. 5,17,28), inactivation of the Pex5 gene by Cre-mediated recombination in oligodendrocytes occurs before myelination, but the exact time point when peroxisome function is lost remains difficult to determine in vivo. In dissociated oligodendrocytes, catalase staining was no longer punctate-that is, associated with peroxisomes-no later than $12 \mathrm{~d}$ in vitro, corresponding approximately to postnatal day 12 or earlier in vivo.

We have shown that peroxisomes are essential for mature oligodendrocytes to maintain the long-term integrity of the subcortical white matter. In addition, the infiltration of $\mathrm{B}$ and $\mathrm{T}$ cells indicates that normal oligodendrocytes may use peroxisomes to maintain a 'noninflammatory' environment within CNS fiber tracts. The relative contributions of specific peroxisomal reaction(s) can now be determined by more specific gene-targeting experiments that separate metabolic functions from the detoxification of ROS or lipid mediators of inflammation ${ }^{29}$. Notably, not all CNS regions were equally affected at the time of premature death. We do not know the reason for this regional heterogeneity, but higher activity of fiber tracts would be a plausible risk factor for earlier degeneration and demyelination. It could explain why mice (relying on olfaction) show neurodegeneration first in the frontal corpus callosum. In humans, relying more on vision, boys with a similarly severe peroxisomal disease, X-linked adrenoleukodystrophy (X-ALD, see below), first show MRI-detectable brain lesions predominantly in the parieto-occipital white matter ${ }^{30-32}$.

Human X-ALD (OMIM 300100) is caused by mutations of the $A B C D 1$ gene, which is ubiquitously expressed and encodes a peroxisomal transporter ${ }^{33,34}$. Whether ABCD1 imports VLCFA into the peroxisome (discussed in ref. 34) remains to be proven. To create a model of X-ALD, we and others have previously generated $A b c d 1$ null mutant mice ${ }^{22-24}$. Unexpectedly, these mutants failed to show inflammatory subcortical demyelination, the main clinical phenotype of $\mathrm{X}$-ALD. Peroxisomal functions are clearly more impaired in the absence of PEX5 than in the absence of ABCD1. It is thus intriguing that Pex5 mutant mice showed virtually all the clinical and histological features of cerebral X-ALD, whereas Abcd1 mutant mice did not (or did only to a very minor extent) ${ }^{35}$. As $A b c d 1$ and Pex5 mutants showed a very similar increase in VLCFA (this study and refs. 22-24), the inflammatory demyelination, as described only in the present study, is unlikely to be caused by the accumulation of VLCFA.

Instead, the paradox between two mutant phenotypes can be explained by ABCD1-deficient peroxisomes that accumulate as-yetunidentified secondary impairments, over a long time ( $>4$ years in humans) and in a cell type-specific fashion. Thus, peroxisomes remain 'functional' in nearly all cells of X-ALD patients, except for oligodendrocytes and adrenal cells that (as we propose) gradually lose more peroxisomal functions. Such secondary peroxisomal changes are not obvious in the short-lived mouse. In humans, only by the time that $\mathrm{X}$-ALD becomes symptomatic (that is, after 5-10 years) have glial peroxisomes accumulated sufficient impairments to cause neurodegeneration. This model can explain why human X-ALD is phenocopied rapidly in Pex5 mutant mice, with all cerebral symptoms of X-ALD, including disease onset after myelination, symmetric subcortical demyelination (with characteristic regional and individual variability), severe axonal loss, VLCFA accumulation, gliosis, and neuroinflammation as marked by infiltrating B and T cells ${ }^{36}$. Although this hypothesis can not be experimentally proven, we note that a few human peroxisomal biogenesis disorders also have a late-onset leukodystrophy phenotype $\mathrm{e}^{37}$. Moreover, in brain autopsy material from X-ALD patients and in white matter of aged Abcd1-deficient mice, peroxisomal catalase puncta are quantitatively reduced (C.M.K. et al., unpublished data). A cell type-specific phenotype following a peroxisomal defect has also been described for liver cells ${ }^{38}$. We suggest that such secondary cell-specific effects can be explained by either the heterogeneity of peroxisomes in different tissues, a cell type-specific metabolism (such as high level cholesterol synthesis in oligodendrocytes and adrenal cells), or both.

Our mice have demonstrated that the primary dysfunction of oligodendrocytes can be sufficient to cause a secondary immune response in the white matter. This is relevant for the role of oligodendrocytes in multiple sclerosis, where myelin is targeted by autoreactive $\mathrm{T}$ cells. The disease relevance of the different $\mathrm{T}$-cell classes 
is still under debate, and the pathogenic role of $\mathrm{CD}^{+}$cells in multiple sclerosis has only recently received increased attention ${ }^{39}$. Moreover, it has been suggested that in some forms of multiple sclerosis, oligodendrocytes show primary pathological changes ${ }^{40}$, but there is no convincing animal model for a primary oligodendrocyte-mediated inflammatory demyelination. B- and T-cell infiltration in Pex5 mutants provides such a model, as it exceeds the bystander effects observed in mice with neurodegeneration ${ }^{41}$ and our previous finding of $\mathrm{CD}^{+} \mathrm{T}$ cells in brains of $P l p 1$ transgenic mice ${ }^{42}$. Characterizing the on molecular diversity of the infiltrating $\mathrm{B}$ and $\mathrm{T}$ cells is an important future goal. By cross-breeding Pex5 conditional to Rag1 mutant mice (which lack functional $\mathrm{B}$ and $\mathrm{T}$ cell receptors) it should be possible to determine the contribution of activated $\mathrm{CD} 8^{+} \mathrm{T}$ cells to the reported demyelinating phenotype. Whether the presence of myelin-specific $\mathrm{T}$ cells is sufficient to turn neuroinflammation, as described here, into more severe inflammation with a self-perpetuating loss of oligodendrocytes can also be tested in this new disease model.

\section{METHODS}

Mouse genetics. The generation of floxed Pex5 and Cnp-Cre mice has been described $^{5,15}$. For genotyping, we isolated genomic DNA from tail biopsies, using the DNeasy96 tissue kit (Qiagen), according to the manufacturer's directions. For routine genotyping, we used PCR primers in a coamplification reaction. All primer sequences are available upon request. Imported Pex $5^{\text {flox }}$ mice were embryo-derived and all mice were maintained in individually ventilated cages under specific pathogen-free conditions. Animal experiments were carried out in compliance with approved animal policies of the Max Planck Institute of Experimental Medicine.

Clinical analysis. Mice were observed monthly. All experiments were conducted by the same investigator and in a blinded fashion. Neurological signs were quantified using a clinical scale with stages I (healthy), II (slight hind limb ataxia), III (severe hind limb ataxia and hunchback), IV (hindlimb paresis and intention tremor) and V (moribund with hind limb paralysis, intention tremor, forelimb ataxia, passiveness and breathing problems). To detect subtle signs of motor coordination defects, we used a grid test. Movements were scored as atactic when mice slipped on the $2-\mathrm{cm}$ grid more than three times within a (2) 100-cm walking distance. Severe ataxia, due to hind limb weakness, was assessed when mice failed to hold on to a round bar $(1.2-\mathrm{cm}$ diameter).

MRI. We carried out high-resolution three-dimensional MRI in vivo at $2.35 \mathrm{~T}$ using a MRBR 4.7/400 mm magnet (Magnex Scientific) equipped with BGA-20 gradient sets driven by a DBX system (Bruker BioSpin MRI GmbH) as described earlier ${ }^{43}$. In brief, mice were intubated and kept under anesthesia (1.0-1.5\% halothane in 70:30 $\mathrm{N}_{2} \mathrm{O}: \mathrm{O}_{2}$ ) with their heads firmly fixed in a purpose-built stereotaxic device. Radio frequency excitation and signal reception were accomplished with use of a Helmholtz coil $(100 \mathrm{~mm})$ and an elliptical surface coil (20 mm anterior-posterior, $12 \mathrm{~mm}$ left-right), respectively. Highresolution datasets at $117-\mu \mathrm{m}^{3}$ isotropic resolution were acquired using a T1-weighted three-dimensional fast low-angle shot (FLASH) MRI sequence (repetition time $17 \mathrm{~ms}$, echo time $7.6 \mathrm{~ms}$, flip angle $25^{\circ}$, accumulations 32 ) and a T2-weighted three-dimensional fast spin-echo MRI sequence (repetition time 3,000 ms, effective echo time $98 \mathrm{~ms}$, accumulations 2).

Histology and electron microscopy. To visualize myelinated fibers, we stained paraffin-embedded tissue sections $(5 \mu \mathrm{m})$ by the Gallyas silver impregnation method ${ }^{44}$. Bielschowsky-Gros silver impregnation ${ }^{45}$ was performed to visualize axons. For TUNEL staining of apoptotic cells, we used the DeadEnd Colorimetric TUNEL System (Promega) according to the manufacturer's directions.

Immunostaining of paraffin sections was visualized using the Dako LSAB2 system according to the manufacturer's directions and analyzed by brightfield light microscopy (Zeiss Axiophot). For fluorescence analysis of immunostained sections, we followed standard procedures, using a confocal laser-scanning microscope (LSM 510, Axiovert 200, Zeiss). For electron microscopy, we perfused mice with $2 \%$ glutaraldehyde and $4 \%$ PFA in PBS. Tissues were postfixed in $1 \% \mathrm{OsO}_{4}$ in $0.1 \mathrm{M}$ sucrose, and embedded in epoxy resin (Serva).
Ultrathin sections were stained for contrast with uranylacetate before examination (EM10, Leo and Zeiss EM 900).

Immunocytochemistry and confocal imaging. Oligodendrocytes from newborn mice were prepared and when differentiated fixed as previously described $^{5}$. Secondary antibodies were conjugated to Cy2 or Cy3 (Molecular Probes, Jackson ImmunoResearch). Fluorescently immunostained sections and cells were visualized with a confocal laser-scanning microscope (LSM 510, Axiovert 200, Zeiss). To detect Cy2 and Alexa Fluor 488 the samples were excited at $488 \mathrm{~nm}$ and detected through a $500-550 \mathrm{~nm}$ bandpass filter. Cy3 was excited at $543 \mathrm{~nm}$ and detected with a $565-615 \mathrm{~nm}$ bandpass filter. All confocal images were processed with the Zeiss LSM software.

Antibodies. We used antibodies at the following concentrations: catalase (Rockland) 1:2,000; CNP (Sigma) 1:300; Cre (Babco) 1:500; MBP (Chemicon) 1:500; antibody (A431) to PLP (polyclonal), 1:600 (ref. 46); PMP-70 (Abcam) 1:600; APP (Chemicon) 1:750 ; GFAP (Chemicon) 1:500; MAC-3 (Pharmingen) 1:100; CD45R (Santa Cruz) 1:100; CD-3 (Serotec) 1:150. Fluorescently labeled secondary antibodies were used as follows: Cy2 (Jackson ImmunoResearch) 1:200; Cy3 (Jackson ImmunoResearch) 1:2,000; Alexa Fluor 488 (Molecular Probes) 1:2,000. For FACS we used antibodies to CD4, CD8 and CD11b from eBiosciences.

Myelin and lipid analysis. Myelin membranes were enriched from total brain lysates by a procedure modified from a published source ${ }^{47}$. Lipid extractions were performed as previously described ${ }^{48}$. After solvent evaporation, samples were resuspended either in $5 \mathrm{mM}$ ammonium acetate in methanol (positive ion mode) or in chloroform-methanol (1:2) (negative ion mode). Nanoscale electrospray ionization tandem mass spectrometry analysis was performed on a Micromass QII triple-stage quadrupole tandem mass spectrometer, equipped with a nano-electrospray ionization source ( $\mathrm{Z}$ spray) from Micromass. Argon was used as collision gas at a nominal pressure of $2.5 \times 10^{-3} \mathrm{mbar}$. The cone voltage was set to 30-50 V. Resolution of Q1 and Q3 was set to achieve isotope resolution. Detection of phosphatidylcholine and sphingomyelin was performed by parent ion scanning for fragment ion $\mathrm{m} / \mathrm{z} 184$ (positive ion mode) at collision energy $32 \mathrm{eV}$; hexosylceramide scanning was performed by parent ion scanning for fragment ion 264 (positive ion mode) at a collision energy of $35 \mathrm{eV}$. Quantification of phosphatidylethanolamine and phosphatidylserine was performed by neutral loss scanning, selecting for a neutral loss of 141 or 185 (positive ion mode), respectively, with a collision energy of $20 \mathrm{eV}$. Phosphatidylethanolamine plasmalogen quantification was performed by parent ion scanning for fragment ion $\mathrm{m} / \mathrm{z} 196$ (negative ion mode; collision energy of $40 \mathrm{eV}$ ). Unsaturated phosphatidylethanolamine and PS standards were synthesized and purified via HPLC as described ${ }^{49}$. VLCFA were determined by gas chromatography/mass spectrometry in a routine clinical diagnostic lab (University of Göttingen).

Quantitative real-time PCR. We prepared lymphocytes and monocytes from brain and peripheral blood by standard procedures, involving Percoll gradients and FACS analysis, as described ${ }^{50}$. RNA was isolated from freshly prepared mouse brains and from sorted cells using the RNeasy Mini and Micro Kits (QIAGEN) following the manufacturer's instructions. We treated samples with DNase I (Roche Diagnostics Corp.), and transcribed $1 \mu \mathrm{g}$ of total brain RNA (or all RNA from FACS sorted cells) into cDNA using oligo(dT) primers and the SuperScript II (or III) RT kit (Invitrogen). For PCR, cDNA (2.5 $\mu$ l) was transferred into a 96-well Multiply PCR plate (Sarstedt), and $12.5 \mu$ l ABsolute

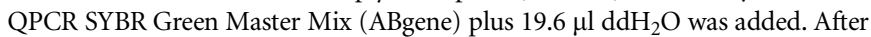
a short Taq polymerase activation step at $95^{\circ} \mathrm{C}$, followed by $45 \mathrm{~s}$ at $63^{\circ} \mathrm{C}$, PCR was started and amplicon accumulation was measured during the annealing phases. We monitored 45 PCR cycles in an iCycler (with data analysis by iCycler analysis software version 2.3; Bio-Rad). The efficiency of each primer was at least $90 \%$, based on the amplification efficiency in serial dilutions. The expression of Gapdh was used as internal standard. Primer sequences are available upon request.

Statistical analysis. To determine significance of mass spectrometric lipid data and quantitative RT-PCR data, we used a two-tailed Student's $t$-test. 
Note: Supplementary information is available on the Nature Genetics website.

\section{ACKNOWLEDGMENTS}

We dedicate this paper to Hugo Moser for his pioneering work on peroxisomes in childhood neurological diseases. We thank J. Barth, U. Bode, A. Fahrenholz, A. Nave, S. Relitz and S. Hühold for excellent technical assistance, and gratefully acknowledge J. Gärtner and D.H. Hunneman for clinical diagnostic service (VLCFA). This work was funded by grants from the European Union (PEX and X-ALD), the US National Multiple Sclerosis Society, the Hertie Foundation, and the generous support of the private Liley and Del Marmol Foundations.

\section{AUTHOR CONTRIBUTIONS}

C.M.K. performed the analyses and drafted the manuscript. C.L.-S. provided Cnp-Cre and M.B. provided Pex5 floxed mice. B.B. determined myelin lipids by mass spectroscopy. A.M. and M.P. performed FACS analysis and quantified cytokines. H.B.W. participated in mouse genetics. O.N., T.M. and J.F. performed MRI. K.-A.N. designed the study and finalized the manuscript.

\section{COMPETING INTERESTS STATEMENT}

The authors declare no competing financial interests.

\section{Published online at http://www.nature.com/naturegenetics}

Reprints and permissions information is available online at http://npg.nature.com/ reprintsandpermissions

1. Lazzarini, R.A. Myelin Biology and Disorders (Elsevier Academic, San Diego, California, USA, 2004).

2. Bjartmar, C. \& Trapp, B.D. Axonal and neuronal degeneration in multiple sclerosis: mechanisms and functional consequences. Curr. Opin. Neurol. 14, 271-278 (2001).

3. Trapp, B.D. et al. Axonal transection in the lesions of multiple sclerosis. N. Engl. J. Med. 338, 278-285 (1998).

o) 4. Griffiths, I. et al. Axonal swellings and degeneration in mice lacking the major proteolipid of myelin. Science 280, 1610-1613 (1998).

5. Lappe-Siefke, C. et al. Disruption of Cnp1 uncouples oligodendroglial functions in axonal support and myelination. Nat. Genet. 33, 366-374 (2003).

6. Suter, U. \& Scherer, S.S. Disease mechanisms in inherited neuropathies. Nat. Rev. Neurosci. 4, 714-726 (2003).

7. Edgar, J.M. et al. Oligodendroglial modulation of fast axonal transport in a mouse model of hereditary spastic paraplegia. J. Cell Biol. 166, 121-131 (2004).

8. Ferreirinha, F. et al. Axonal degeneration in paraplegin-deficient mice is associated with abnormal mitochondria and impairment of axonal transport. J. Clin. Invest. 113, 231-242 (2004)

9. Schrader, M. \& Fahimi, H.D. Mammalian peroxisomes and reactive oxygen species. Histochem. Cell Biol. 122, 383-393 (2004).

(3) 10. Wanders, R.J. Peroxisomes, lipid metabolism, and peroxisomal disorders. Mol. Genet. Metab. 83, 16-27 (2004).

11. Baes, M. et al. A mouse model for Zellweger syndrome. Nat. Genet. 17, 49-57 (1997)

12. Hirrlinger, J., Resch, A., Gutterer, J.M. \& Dringen, R. Oligodendroglial cells in culture effectively dispose of exogenous hydrogen peroxide: comparison with cultured neurones, astroglial and microglial cells. J. Neurochem. 82, 635-644 (2002).

13. Gould, S.J. \& Collins, C.S. Opinion: peroxisomal-protein import: is it really that complex? Nat. Rev. Mol. Cell Biol. 3, 382-389 (2002).

14. Dodt, G. et al. Mutations in the PTS1 receptor gene, PXR1, define complementation group 2 of the peroxisome biogenesis disorders. Nat. Genet. 9, 115-125 (1995).

15. Baes, M., Dewerchin, M., Janssen, A., Collen, D. \& Carmeliet, P. Generation of Pex5loxP mice allowing the conditional elimination of peroxisomes. Genesis 32, 177-178 (2002).

16. Chang, C.C. et al. Metabolic control of peroxisome abundance. J. Cell Sci. 112 1579-1590 (1999)

17. Saher, G. et al. High cholesterol level is essential for myelin membrane growth. Nat. Neurosci. 8, 468-475 (2005).

18. Zoeller, R.A. \& Raetz, C.R. Isolation of animal cell mutants deficient in plasmalogen biosynthesis and peroxisome assembly. Proc. Natl. Acad. Sci. USA 83, 5170-5174 (1986)

19. Rodemer, C. et al. Inactivation of ether lipid biosynthesis causes male infertility, defects in eye development and optic nerve hypoplasia in mice. Hum. Mol. Genet. 12, 1881-1895 (2003).

20. Moser, H.W., Bergin, A. \& Cornblath, D. Peroxisomal disorders. Biochem. Cell Biol. 69, 463-474 (1991).

21. Wanders, R.J. \& Waterham, H.R. Peroxisomal disorders I: biochemistry and genetics of peroxisome biogenesis disorders. Clin. Genet. 67, 107-133 (2005).
22. Forss-Petter, S. et al. Targeted inactivation of the X-linked adrenoleukodystrophy gene in mice. J. Neurosci. Res. 50, 829-843 (1997).

23. Lu, J.F. et al. A mouse model for X-linked adrenoleukodystrophy. Proc. Natl. Acad. Sci. USA 94, 9366-9371 (1997).

24. Kobayashi, T., Shinnoh, N., Kondo, A. \& Yamada, T. Adrenoleukodystrophy proteindeficient mice represent abnormality of very long chain fatty acid metabolism. Biochem. Biophys. Res. Commun. 232, 631-636 (1997).

25. Simpson, J.E., Newcombe, J., Cuzner, M.L. \& Woodroofe, M.N. Expression of monocyte chemoattractant protein-1 and other beta-chemokines by resident glia and inflammatory cells in multiple sclerosis lesions. J. Neuroimmunol. 84, 238-249 (1998).

26. McManus, C. et al. MCP-1, MCP-2 and MCP-3 expression in multiple sclerosis lesions: an immunohistochemical and in situ hybridization study. J. Neuroimmunol. 86, 20-29 (1998).

27. Loers, G., Aboul-Enein, F., Bartsch, U., Lassmann, H. \& Schachner, M. Comparison of myelin, axon, lipid, and immunopathology in the central nervous system of differentially myelin-compromised mutant mice: a morphological and biochemical study. Mol. Cell. Neurosci. 27, 175-189 (2004).

28. Genoud, S. et al. Notch1 control of oligodendrocyte differentiation in the spinal cord. J. Cell Biol. 158, 709-718 (2002).

29. Schrader, M. \& Fahimi, H.D. Peroxisomes and oxidative stress. Biochim. Biophys. Acta 1763, 1755-1766 (2006).

30. Loes, D.J. et al. Analysis of MRI patterns aids prediction of progression in X-linked adrenoleukodystrophy. Neurology 61, 369-374 (2003).

31. Wilken, B. et al. Quantitative proton magnetic resonance spectroscopy of children with adrenoleukodystrophy before and after hematopoietic stem cell transplantation. Neuropediatrics 34, 237-246 (2003).

32. Dubois-Dalcq, M., Feigenbaum, V. \& Aubourg, P. The neurobiology of X-linked adrenoleukodystrophy, a demyelinating peroxisomal disorder. Trends Neurosci. 22, 4-12 (1999).

33. Mosser, J. et al. Putative X-linked adrenoleukodystrophy gene shares unexpected homology with ABC transporters. Nature 361, 726-730 (1993).

34. Kemp, S. \& Wanders, R.J. X-linked adrenoleukodystrophy: Very long-chain fatty acid metabolism, ABC half-transporters and the complicated route to treatment. Mol. Genet. Metab. 90, 268-276 (2007).

35. Pujol, A. et al. Late onset neurological phenotype of the X-ALD gene inactivation in mice: a mouse model for adrenomyeloneuropathy. Hum. Mol. Genet. 11, 499-505 (2002).

36. Hudspeth, M.P. \& Raymond, G.V. Immunopathogenesis of adrenoleukodystrophy: current understanding. J. Neuroimmunol. 182, 5-12 (2006).

37. Barth, P.G. et al. Late onset white matter disease in peroxisome biogenesis disorder. Neurology 57, 1949-1955 (2001).

38. Baumgartner, M.R. et al. Clinical approach to inherited peroxisomal disorders: a series of 27 patients. Ann. Neurol. 44, 720-730 (1998).

39. Goverman, J. et al. The role of $\mathrm{CD} 8^{+} \mathrm{T}$ cells in multiple sclerosis and its animal models. Curr. Drug Targets Inflamm. Allergy 4, 239-245 (2005).

40. Barnett, M.H. \& Prineas, J.W. Relapsing and remitting multiple sclerosis: pathology of the newly forming lesion. Ann. Neurol. 55, 458-468 (2004).

41. Raivich, G. et al. Immune surveillance in the injured nervous system: T-lymphocytes invade the axotomized mouse facial motor nucleus and aggregate around sites of neuronal degeneration. J. Neurosci. 18, 5804-5816 (1998).

42. Ip, C.W. et al. Immune cells contribute to myelin degeneration and axonopathic changes in mice overexpressing proteolipid protein in oligodendrocytes. J. Neurosci. 26, 8206-8216 (2006).

43. Natt, 0 . et al. High-resolution 3D MRI of mouse brain reveals small cerebral structures in vivo. J. Neurosci. Methods 120, 203-209 (2002).

44. Gallyas, F. Silver staining of myelin by means of physical development. Neurol. Res. 1, 203-209 (1979).

45. Bielschowsky, M. Die Silberimprägnation der Neurofibrillen. J. Psychol. Neurol. 3, 169-183 (1904).

46. Jung, M., Sommer, I., Schachner, M. \& Nave, K.A. Monoclonal antibody 010 defines a conformationally sensitive cell-surface epitope of proteolipid protein (PLP): evidence that PLP misfolding underlies dysmyelination in mutant mice. J. Neurosci. 16, 7920-7929 (1996).

47. Norton, W.T. \& Poduslo, S.E. Myelination in rat brain: method of myelin isolation. J. Neurochem. 21, 749-757 (1973).

48. Bligh, E.G. \& Dyer, W.J. A rapid method of total lipid extraction and purification. Can. J. Biochem. Physiol. 37, 911-917 (1959).

49. Koivusalo, M., Haimi, P., Heikinheimo, L., Kostiainen, R. \& Somerharju, P. Quantitative determination of phospholipid compositions by ESI-MS: effects of acyl chain length, unsaturation, and lipid concentration on instrument response. J. Lipid Res. 42, 663-672 (2001).

50. Gutcher, I., Urich, E., Wolter, K., Prinz, M. \& Becher, B. Interleukin 18-independent engagement of interleukin 18 receptor is required for autoimmune inflammation. Nat. Immunol. 7, 946-953 (2006). 